\title{
Beitrag zur Kenntnis des Tryptophans und einiger seiner Derivate. \\ Von
}

Emil Abderhalden und Martin Kempe.

(Aus dem I. chemischen Institut der Universität Berlin.)

(Der Redaktion zugegangen am 25. Mai 1907.)

Durch die Untersuchungen von Hopkins und Cole ${ }^{1}$ ) ist das Tryptophan ein verhältnismäßig leicht zugängliches Spaltprodukt vieler Eiweißkörper geworden. Man wird ihm ohne Zweifel noch recht oft begegnen. Es ist wünschenswert, zu seiner Isolierung und Reinigung eine Reihe von charakteristischen Derivaten zu kennen. Vielleicht ermöglicht das eine oder andere Derivat eine quantitative Bestimmung des Tryptophans. Es sind bereits mehrere Verbindungen des Tryptophans eingehend beschrieben worden, so von Hopkins und Cole das schwerlösliche salzsaure Salz des Tryptophans, ferner haben N euberg und Popowsky²) den bei der Einwirkung von Brom- resp. Chlorwasser auf eine wässerige Tryptophanlösung sich bildenden Farbstoff genauer untersucht und die folgenden Verbindungen isoliert und beschrieben: $\mathrm{C}_{11} \mathrm{H}_{11} \mathrm{~N}_{2} \mathrm{O}_{2} \mathrm{Br}, \mathrm{C}_{11} \mathrm{H}_{11} \mathrm{~N}_{2} \mathrm{O}_{2} \mathrm{Br} \cdot \mathrm{Br}_{2}$, $\mathrm{C}_{11} \mathrm{H}_{11} \mathrm{~N}_{2} \mathrm{O}_{2} \mathrm{Cl}$ und $\mathrm{C}_{11} \mathrm{H}_{11} \mathrm{~N}_{2} \mathrm{O}_{2} \mathrm{Cl} \cdot \mathrm{Cl}_{2}$. SchlieBlich hat neuerdings M. Mayeda ${ }^{8}$ ) das Pikrat und Pikrolonat des Tryptophans dargestellt. Wir haben folgende Verbindungen gewonnen: 1. das Kupfersalz des Tryptophans, 2. das d-Tryptophanmethylesterchlorhydrat, 3. den d-Tryptophanmethylester, 4. Phenylisocyanat-d-Tryptophan, 5. das Natriumsalz des $\beta$-Naph talinsulfo-d-Tryptophans und endlich das salzsaure Tryptophanchlorid.

1) F. G. Hopkins u. S. W. Gole, A contribution to the chemistry of proteids. J. of Physiol., Bd. XXVII, S. 418, 1901.

2) G. Neuberg und N. Popowsky, Die Indolaminopropionsäure und ihre Halogenverbindungen (Tryptophanreaktion). Biochem. Zeitschrift, Bd. II, S. 357, 1907.

3) M. Mayeda, Zum Nachweis des Tryptophans und des Phenylalanins. Diese Żeitschrift, Bd. LI, S. 261, 1907. 
Das Tryptophan, das zu diesen Versuchen diente, hatten wir im wesentlichen nach der Vorschrift von Hopkins und Cole dargestellt.

$5 \mathrm{~kg}$ Casein wurden in $50 \mathrm{l} \mathrm{0,8 \%} /$ iger Sodalösung aufgeschwemmt, mit $20 \mathrm{~g}$ Pankreatin gut durchgerührt und die Mischung nach Zugabe einer ausreichenden Menge von Toluol bei einer Temperatur von $36^{\circ}$ aufbewahrt. Von Zeit zu Zeit prüften wir in Proben mit Bromwasser auf freies Tryptophan. Nachdem die Tryptophanreaktion einen Maximalwert erreicht hatte, was nach 5-10 Tagen der Fall war, wurde die Verdauung unterbrochen, das Gemisch auf $80^{\circ}$ erwärmt, um noch vorhandenes Eiweiß zum Koagulieren zu bringen. Es empfiehlt sich, die Lösung in der Hitze mit einer reichlichen Menge reiner Infusorienerde durchzurühren. Es erleichtert dies das spätere Filtrieren erheblich. Beim Abkühlen der Flüssigkeit schied sich Tyrosin in reichlichen Mengen aus. Die abgekühlte Flüssigkeit filtrierten wir nun durch Koliertuch und versetzten das klare Filtrat mit 5 Volumenprozent Schwefelsäure und einem Überschuß einer 10\% igen Quecksilbersulfatlösung in 5 volumprozentiger Schwefelsäure. Es entstand bald ein reichlicher Niederschlag. Er wurde nach 12stündigem Stehen abfiltriert, der scharf abgepreßte Filterrückstand mit 5\% iger Schwefelsäure gründlich ausgewaschen, dann in Wasser suspendiert und mit Schwefelwasserstoff unter öfterer Erwärmung und kräftigem Rühren mit einer Turbine zersetzt. Nach dem Filtrieren wurde der Filterrückstand noch zweimal mit Wasser verrieben und in die Suspension Schwefelwasserstoff eingeleitet, um so noch unzersetztes Quecksilbersalz zu zerlegen.

Aus den vereinigten Filtraten entfernten wir den Schwefelwasserstoff nicht durch Kochen, sondern durch einen kräftigen Kohlensäurestrom. Durch Erwärmen entstehen zu leicht verharzende Produkte. Sobald kein Geruch nach Schwefelwasserstoff mehr wahrnehmbar war, versetzten wir die Lösung wiederum mit 5\% ihres Volumens an Schwefelsäure und mit soviel Quecksilbersulfatlösung, daß sich gerade ein zusammenhängender Niederschlag bildete. Diesen filtrierten wir nach einer halben Stunde ab. Es gelingt so, das zuerst ausfallende Cystin und 
ferner etwa vorhandene Verharzungsprodukte zu entfernen. Das Filtrat vom Niederschlage wurde nun mit überschüssigem Quecksilbersulfat versetzt, der entstandene Niederschlag abfiltriert, und dieser wie das erstemal mit Schwefelwasserstoff zerlegt. Aus der hierbei gewonnenen Lösung fällten wir die Schwefelsäure quantitativ mit Barythydrat.

Die vom Baryumsulfat abfiltrierte Flüssigkeit war nur noch schwach gelb gefärbt. Nach der Vorschrift von Hopkins und Cole soll die das Tryptophan enthaltende Lösung nunmehr auf dem W'asserbad unter häufigem Zusatz von Alkohol eingedampft werden. Hierbei tritt jedoch sehr leicht Verharzung ein, wodurch die Ausbeute an reinem Material stark beeinträchtigt werden kann. Sie läßt sich vollständig vermeiden, wenn man das Eindampfen unter vermindertem Druck vornimmt. Die neutrale Lösung wurde nach Zusatz von wenig Alkohol, um das Schäumen $\mathrm{zu}$ vermeiden, bei etwa $14 \mathrm{~mm}$ Druck und einer $40^{\circ}$ des Wasserbades nicht übersteigenden Temperatur soweit eingedampft, bis sich eine reichliche Menge Tryptophan ausgeschieden hatte. Von diesem wurde abfiltriert und die Mutterlauge weiter eingeengt, bis noue Krystallmassen zur Abscheidung kamen. Wir erhielten so noch mehrere Fraktionen. Das so gewonnene Tryptophan war farblos. Die Ausbeute betrug 26,5 g Rohprodukt. Dieses lieferte nach dem Umkrystallisieren aus verdünntem Alkohol unter Zusatz von Tierkohle $24 \mathrm{~g}$ analysenreines Produkt.

$0,2201 \mathrm{~g}$ Substanz bei $100^{\circ}$ getrocknet gaben $0,5199 \mathrm{~g} \mathrm{CO}_{\mathrm{z}}$ und $0,1178 \mathrm{~g} \mathrm{H}_{2} \mathrm{O}$.

$0,1365 \mathrm{~g}$ Substanz bei $100^{\circ}$ getrocknet gaben $15,9 \mathrm{ccm} \mathrm{N}$ bei $18^{\circ}$ und $753 \mathrm{~mm}$ Druck.

Berechnet für $\mathrm{C}_{11} \mathrm{H}_{12} \mathrm{~N}_{2} \mathrm{O}_{8}(204,2): 64,64 \% \mathrm{C}, 5,92 \% \mathrm{H}, 13,75 \% \mathrm{~N}$. Gefunden: $\quad 64,42 \% \mathrm{C}, 5,99 \% \mathrm{H}, 13,54 \% \mathrm{~N}$.

Die angegebene Ausbeute wurde nicht bei jeder Darstellung erreicht. Besonders bei langer Verdauung und langsamer Verarbeitung blieb sie oft hinter diesem Betrage zurück. In diesen Fällen gelang es, ein Nebenprodukt zu fassen, das weiter unten beschrieben ist.

Das auf die geschilderte Art gewonnene Tryptophan prüften wir auf sein optisches Verhalten. Hopkins und Cole geben als spezifische Drehung des Tryptophans den Wert $\left[\alpha_{D}\right]=-33^{\circ}$ 
an. Leider fehlt die Angabe, unter welchen Verhältnissen und mit welchem Lösungsmittel sie gearbeitet haben.

C. Neuberg und N. Popowsky ${ }^{1}$ ) geben folgende Werte für das Tryptophan an: in wässeriger Lösung $\left[\alpha_{D}\right]=-7,8^{\circ}$, in $1 / 2$ normaler Natronlauge $\left[\alpha_{D}\right]=-13,7^{\circ}$.

Das wie oben beschrieben dargestellte Tryptophan dreht im Gegensatz zu diesen Angaben nach rechts.

Eine Lösung von 0,2186 $\mathrm{g}$ Tryptophan, das viermal umkrystallisiert war, in $1 / 2$ normaler Natronlauge vom Gesamtgewicht 7,9913 $\mathrm{g}$ und dem spezifischen Gewicht 1,02 drehte im $1 \mathrm{dm}-$ Rohr bei Natriumlicht und $20^{\circ}$ C. $0,16^{\circ}$ nach rechts. Mithin

$$
[\alpha]_{D}^{20}=+5,7^{\circ}
$$

Wir waren in der glücklichen Lage, ein Präparat unter denselben Bedingungen prüfen zu können, das wir der Liebenswürdigkeit von Herrn Prof. Hopkins verdanken.

$0,1062 \mathrm{~g}$ dieses Präparates wurden in ${ }^{1 / 2}$ normaler Natronlauge gelöst. Gesamtgewicht der Lösung $4,0650 \mathrm{~g}$; spezifisches Gewicht 1,03. Drehung im $1 \mathrm{dm}-$ Rohr bei Natriumlicht und $20^{\circ}$ G. $0,17^{\circ}$ nach rechts. Mithin

$$
[\alpha]_{D}^{20}=+6,3^{\circ} \text {. }
$$

Beide Präparate zeigten somit ein ganz ähnliches optisches Verhalten. Es muß weiteren Untersuchungen vorbehalten bleiben, festzustellen, auf welchen Ursachen das ganz andere Verhalten des von Neuberg und Popowsky untersuchten Präparates beruht.

Weitere Bestimmungen führten wir in normaler Natronlauge aus.

$0,6043 \mathrm{~g}$ viermal umkrystallisiertes Tryptophan wurden in normaler Natronlauge gelöst. Gesamtgewicht der Lösung $5,3108 \mathrm{~g}$. Spezifisches Gewicht 1,06. Drehung im $1 \mathrm{dm}-\mathrm{Rohr}$ bei Natriumlicht und $20^{\circ}$ C. $0,74^{\circ}$ nach rechts. Mithin

$$
[\alpha]_{\mathrm{D}}^{20}=+6,12^{\circ} \text {. }
$$

Dasselbe Präparat wurde nach nochmaligem Umkrystallisieren untersucht.

1) l. c. 
Beitrag zur Kenntnis des Tryptophans und einiger seiner Derivate. 211

$0,4543 \mathrm{~g}$ Tryptophan wurden in normaler Natronlauge gelöst. Gesamtgewicht der Lösung 4,6305 g. Spezifisches Gewicht 1,06. Drehung im $1 \mathrm{dm}-\mathrm{Rohr}$ bei Natriumlicht und $20^{\circ} \mathrm{C}$. $0,63^{\circ}$ nach rechts. Mithin

$$
[a]_{\mathrm{D}}^{20}=+6,06^{\circ} \text {. }
$$

Die Drehung blieb somit konstant. Mit demselben Präparat wurde auch noch eine Bestimmung in salzsaurer Lösung vorgenommen. 0,4517 g Tryptophan wurden in normaler Salzsäure gelöst. Gesamtgewicht der Lösung $7,2161 \mathrm{~g}$; spezifisches Gewicht 1,03. Drehung im 2-dm-Rohr bei $20^{\circ} \mathrm{C}$. und Natriumlicht $0,17^{\circ}$ nach rechts. Mithin

$$
[\alpha]_{\mathrm{D}}^{20}=+1,31^{\circ}
$$

Das Tryptophan schmilzt gegen $289^{\circ}$ (korr.). Von $260^{\circ}$ (korr.) an zeigt sich leichte Gelbfärbung.

Es sei noch erwähnt, daß das dargestellte Tryptophan alle beschriebenen Farbenreaktionen gab. Vor allem beobachteten wir auch die von Cole ${ }^{1}$ ) beschriebene Violettfärbung beim Kochen von Tryptophan mit Zucker und rauchender Salzsäure, eine Färbung, welche der bei der sog. Liebermannschen Reaktion auftretenden sehr ähnlich ist. Es ist möglich, daß die Liebermannsche Reaktion auf einer ähnlichen Ursache beruht, d. h. auf das Vorhandensein von Tryptophan und Zucker hinweist. Sicher festgestellt ist diese Deutung der genannten Reaktion nicht, gibt doch z. B. Seide, unter dessen Spaltprodukten Tryptophan bis jetzt nicht aufgefunden worden ist, ebenfalls beim Lösen in konzentrierter Salzsäure Violettfärbung und zwar besonders ausgeprägt in der Kälte. Es scheinen übrigens nicht alle Zuckerarten gleich gut geeignet zu sein. Eine ausgeprägte Violettfärbung erhielten wir nur bei Verwendung von Fruktose, Rohrzucker und Raffinose. Glukose, Galaktose und Mannose gaben die Reaktion nur schwach oder undeutlich. Die Pentosen, Arabinose und Xylose verursachten nur eine schmutzig braune Färbung.

Nicht ohne Interesse ist die Beobachtung, daß Trypto-

i) Sydney W. Cole, On certain colour reactions of proteid due to tryptophane. Journal of Physiol., Bd. XXX, S. 311, 1903. 
phan in verdünnter wässeriger Lösung beim Kochen mit Salpetersäure deutlich Gelbfärbung zeigt. Ferner gibt Tryptophan beim Kochen mit dem sog. Millonschen Reagens Braunrotfärbung. Diese Reaktion ist sehr wohl von der bei Anwesenheit von Tyrosin auftretenden Färbung zu unterscheiden. Es dürfte jedoch nicht überflüssig sein, um Täuschungen zu vermeiden, auf diese Reaktion hinzuweisen.

Isolierung eines Nebenproduktes bei der Tryptophandarstellung.

Wie oben erwähnt, gelang es in einigen Fällen, bei denen die Ausbeute an Tryptophan nur gering war, statt dessen einen anderen Körper zu isolieren. Dies fand dann statt, wenn die Verdauung des Caseins sehr lange gewährt hatte, und die Verarbeitung nur sehr langsam erfolgt war. Beim Eindampfen der tryptophanhaltigen Lösung unter vermindertem Druck schied sich dann als erste Fraktion ein Produkt aus, das schwach gelb gefärbt war und in Nadeln krystallisierte, während das in farblosen Blättchen krystallisierende Tryptophan in den späteren Fraktionen enthalten war.

Das neue Produkt ist in Wasser noch schwerer löslich als das Tryptophan. Es wurde durch mehrfaches Umkrystallisieren aus heißem Wasser unter Zusatz von Tierkohle gereinigt. Es schmilzt bei $293^{\circ}$ (korr.), nachdem es vorher gegen $276^{\circ}$ (korr.) angefangen hat, sich gelb $\mathrm{zu}$ färben. Aus der wässerigen Lösung scheidet es sich in büschelförmig vereinigten Nadeln ab. Zur Analyse wurde es im Vakuum über Phosphorpentoxyd bei $140^{\circ}$ getrocknet. Die Verbrennung ergab Werte, die der Formel $\mathrm{C}_{11} \mathrm{H}_{12} \mathrm{~N}_{2} \mathrm{O}_{3}$ entsprechen.

$0,1530 \mathrm{~g}$ Substanz gaben $0,3348 \mathrm{~g} \mathrm{CO}_{2}$ und $0,0791 \mathrm{~g} \mathrm{H}_{2} \mathrm{O}$.

$0,1207 \mathrm{~g}$ Substanz gaben $13,3 \mathrm{ccm} \mathrm{N}$ bei $18^{\circ}$ und $735 \mathrm{~mm}$ Druck.

Berechnet für $\mathrm{C}_{11} \mathrm{H}_{12} \mathrm{~N}_{2} \mathrm{O}_{3}(220,2): 59,94 \% \mathrm{G}, 5,49 \% \mathrm{H}, 12,75 \% \mathrm{~N}$.

Gefunden: $59,68 \% \mathrm{C}, 5,78 \% \mathrm{H}, 12,50 \% \mathrm{~N}$.

Die Substanz entwickelt beim Erhitzen starken Indol- oder Skatolgeruch. Da sie aus dem Tryptophan höchstwahrscheinlich durch Oxydation hervorgegangen ist, dürfte sie als 0xytryptophan $z u$ bezeichnen sein. Interessant ist es, daß sie nicht mehr die Reaktion auf Tryptophan mit Bromwasser gibt. Da- 
gegen liefert sie beim Erhitzen mit konzentrierter Bromwasserstoffsäure einen violetten Farbstoff. Die Reaktion mit Glyoxylsäure nach Hopkins und Cole gibt das isolierte Produkt nur bei recht vorsichtigem Zusatz von Schwefelsäure zu der mit. Glyoxylsäure versetzten Substanz. Bei Zusatz von zuviel Schwefelsäure findet die Reaktion nicht statt.

Sehr bemerkenswert ist das Verhalten des Körpers nach dem Erhitzen mit konzentrierter Salzsäure. Kocht man den. Körper mit konzentrierter Salzsäure, dampft dann die Lösung ein und erhitzt jetzt den Rückstand, für sich oder mit Natriumhydrat, so ist nach genügend langem Erhitzen kein Indol- oder Skatolgeruch mehr zu bemerken. Statt dessen tritt ein ganz starker, charakteristischer Geruch nach Chinolin auf. Aus Mangel an der nötigen Menge dieses Produktes konnte die nähere Untersuchung dieses interessanten Verhaltens vorläufig noch nicht eingehender vorgenommen werden. Diese Lücke soll jedoch bald ausgefüllt werden.

Im folgenden seien die dargestellten Derivate des Tryptophans beschrieben.

\section{Kupfersalz des Tryptophans.}

Kocht man Tryptophan mit frisch gefälltem, aufgeschlemmtem Kupferoxyd, so erhält man keine blaue Lösung, wie bei den meisten anderen Aminosäuren. Das Tryptophan ist vollkommen in den Kupferoxydniederschlag übergegangen, was daran zu erkennen ist, daß in der abfiltrierten Lösung jede Tryptophanreaktion ausbleibt. Zur Isolierung des Salzes wurde folgender Weg eingeschlagen: $1 \mathrm{~g}$ Tryptophan wurde in wässeriger Lösung mit wenig aufgeschlemmtem Kupferoxyd erst eine Stunde auf dem Wasserbade erwärmt und dann 15 Minuten über freier Flamme gekocht. Nun wurde mit Eiswasser abgekühlt und kalte verdünnte Salzsäure hinzugegeben, um das Kupferoxyd in Lösung zu bringen. Das Kupfersalz des Tryptophans blieb als hellblauer Niederschlag ungelöst. Dieser wurde abfiltriert und mit Wasser bis zum Verschwinden der Halogenreaktion gewaschen. Nach dem Trocknen stellt das Tryptophankupfer ein feines, graublaues Pulver dar. Unter dem Mikroskop sind keine deut- 
lichen Krystalle zu erkennen. Das Tryptophankupfer ist in den gewöhnlichen Lösungsmitteln und in kalten verdünnten Mineralsäuren schwer löslich. Das Ergebnis der Analyse spricht für folgende Zusammensetzung des Kupfersalzes :

$$
\left(\mathrm{C}_{11} \mathrm{H}_{11} \mathrm{~N}_{2} \mathrm{O}_{2}\right)_{2} \mathrm{Cu} \text {. }
$$

Zur Analyse wurde es bei $100^{\circ}$ getrocknet. $0,1718 \mathrm{~g}$ Substanz gaben $0,0285 \mathrm{~g} \mathrm{CuO}$.

Berechnet für $\left(\mathrm{C}_{11} \mathrm{H}_{11} \mathrm{~N}_{2} \mathrm{O}_{2}\right)_{2} \mathrm{Cu}(470): 13,53 \% \mathrm{Cu}$. Gefunden: $\quad 13,25 \% \mathrm{Cu}$.

d-Tryptophan-methylesterchlorhydrat.<smiles>CC(=O)C(CC1CNC2CCCC12)NCl</smiles>

$5 \mathrm{~g}$ Tryptophan wurden mit $50 \mathrm{ccm}$ Methylalkohol übergossen und gasförmige Salzsäure unter Eiskühlung eingeleitet. Zum Schluß wurde auf Zimmertemperatur erwärmt. Die Aminosäure ging dabei völlig in Lösung. Überschüssige Salzsäure und Methylalkohol wurden im Vakuum bei niederer Temperatur abgedampft. Den Rückstand lösten wir in wenig heißem Methylalkohol und gaben allmählich viel heißen Essigester unter Reiben mit dem Glasstab zu. Beim Erkalten schied sich das Chlorhydrat des Tryptophanmethylesters fein krystallinisch aus. Wir erhielten 4,5 $\mathrm{g}$ des Esterchlorhydrates. Durch Verarbeiten der Mutterlauge ließ sich die Ausbeute noch verbessern, doch war die zweite Fraktion bedeutend unreiner als die erste. Durch nochmaliges Lösen in Methylalkohol und Ausfällen mit Essigester erhielten wir den Körper als weißes krystallinisches Pulver. Das Tryptophanmethylesterchlorhydrat löst sich leicht in Alkohol und Wasser, schwer in Essigester und Äther. Aus Methylalkohol mit Essigester gefällt, bildet die Verbindung kleine mikroskopische Nadeln, die meist zu büschelförmigen Aggregaten vereinigt sind. Sie schmilzt gegen $214^{\circ}$ (korr.) unter starker Zersetzung und Gasentwickelung. Zur Analyse wurde die Substanz über Chlorcalcium im Vakuum getrocknet.

$0,2041 \mathrm{~g}$ Substanz gaben $0,4256 \mathrm{~g} \mathrm{CO}_{2}$ und $0,1087 \mathrm{~g} \mathrm{H}_{2} \mathrm{O}$.

$0,1517 \mathrm{~g}$ Substanz gaben $14,6 \mathrm{ccm} \mathrm{N}$ bei $18^{\circ}$ und $753 \mathrm{~mm}$ Druck.

0,1977 g Substanz verbrauchten 7,72 ccm 1/10-normal Silberlösung. 


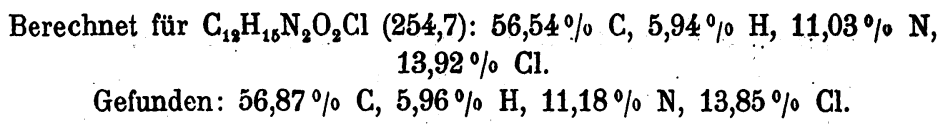

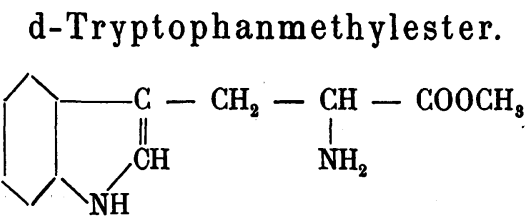

2,3 g Tryptophanmethylesterchlorhydrat wurden mit $40 \mathrm{ccm}$ Äther überschichtet, die Mischung in einer Kältemischung abgekühlt, dann $1 \mathrm{ccm} 10$ fach normale Natronlauge und $5 \mathrm{ccm}$. Wasser hinzugegeben. Nun wurde kräftig durchgeschüttelt und trocknes Kaliumcarbonat hinzugegeben, bis die Masse breiartige Konsistenz erlangte. Der Äther wurde bald abgegossen und durch neuen ersetzt. Dieses Verfahren wiederholten wir, bis. etwa $160 \mathrm{ccm}$ Äther verbraucht waren. Die vereinigten filtrierten ätherischen Auszüge wurden nach 12stündigem Stehen über Natriumsulfat im Vakuum eingedampft. Hierbei schied sich der Ester krystallinisch aus. Er wurde abfiltriert und mit kaltem Äther gewaschen. Die Ausbeute an dieser ersten Fraktion betrug $1,5 \mathrm{~g}$ an farblosem Produkt. Durch Eindampfen der Mutterlauge ließen sich noch $0,25 \mathrm{~g}$ isolieren. Die erste Fraktion war bereits völlig analysenreiner Tryptophanmethylester. Er schmilzt bei $89,5^{\circ}$ (korr.) und ist leicht löslich in Methylalkohol, schwerer in Essigester und Äther, sehr schwer in Petroläther. Aus Äther umkrystallisiert, bildet er ziemlich große Tafeln, die an einem Ende rechtwinklig abgeschnitten, am andern zugespitzt sind: Oft bildet die Verbindung Krusten aus konzentrisch krystallinischen, nierenförmigen Aggregaten. Sie ist recht beständig und läßt sich unzersetzt aufbewahren. Die methylalkoholische und ätherische Lösung zersetzt sich selbst bei langem Stehen im Brutraum nicht. Beim Verseifen mit Alkalien wird Tryptophan zurückgebildet.

Zur Analyse des Tryptophanmethylesters benutzten wir. die bei der oben beschriebenen Darstellung erhaltene erste Fraktion. Die Substanz wurde im Vakuum über Chlorcalcium getrocknet. 
$0,1400 \mathrm{~g}$ Substanz gaben $0,3385 \mathrm{~g} \mathrm{CO}_{2}$ und $0,0813 \mathrm{~g} \mathrm{H}_{2} \mathrm{O}$. $0,2232 \mathrm{~g}$ Substanz gaben $25,3 \mathrm{ccm} \mathrm{N}$ bei $18^{\circ}$ und $746 \mathrm{~mm}$ Druck. Berechnet für $\mathrm{C}_{12} \mathrm{H}_{14} \mathrm{~N}_{2} \mathrm{O}_{2}(218,2): 66,00 \% \mathrm{C}, 6,47 \% \mathrm{H}, 12,87 \% \mathrm{~N}$. Gefunden: $65,94 \% \mathrm{C}, 6,50 \% \mathrm{H}, 13,05 \% \mathrm{~N}$.

Phenylisocyanat-d-Tryptophan.

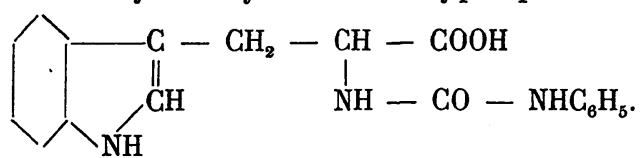

$2 \mathrm{~g}$ Tryptophan wurden in $10 \mathrm{ccm}$ normaler Natronlauge gelöst, die Lösung in einer Kältemischung abgekühlt und allmählich unter Umschütteln mit 1,2 $\mathrm{g}$ Phenylcyanat versetzt. Dann wurde mit $12 \mathrm{ccm}$ normaler Salzsäure angesäuert. Hierbei schied sich die Verbindung amorph ab. Sie wurde abfiltriert und mit Wasser gewaschen. Die Ausbeute betrug nach dem Trocknen im Vakuum 2,2 g. Das Filtrat wurde im Vakuum eingedampft und der Rückstand mit Alkohol ausgezogen. Durch Ausfällen der alkoholischen Lösung mit Wasser ließen sich noch $0,7 \mathrm{~g}$ eines weniger reinen Produktes isolieren. Zur vollständigen Reinigung wurde die neue Verbindung in Methylalkohol gelöst, die Lösung mit Tierkohle erwärmt, dann filtriert und die Verbindung aus dem Filtrat mit Wasser ausgefällt. Sie schied sich dabei in feinen Nadeln ab. Nach zweimaligem Umkrystallisieren zeigte sie den konstanten Schmelzpunkt $166^{\circ}$ (korr.). Sie löst sich leicht in Alkohol, Essigester und Aceton, schwer in kaltem Wasser. Bemerkenswert ist bei dieser Substanz ihre starke Lichtempfindlichkeit. Das ursprünglich farblose Produkt färbt sich im zerstreuten Tageslicht rosa. Setzt man es dem direkten Sonnenlicht aus, so wird es intensiv rot. Auch der Schmelzpunkt scheint durch Belichtung stark beeinflußt zu werden. Ein Präparat, das den Schmelzpunkt $166^{\circ}$ zeigte, wurde an einem hellen Tage umkrystallisiert. Die Substanz zeigte nun plötzlich den Schmelzpunkt $132^{\circ}$. Sie wurde nun noch einmal, aber unter Lichtabschluß umkrystallisiert. Danach stieg der Schmelzpunkt wieder auf den richtigen Wert von $166^{\circ}$. Es empfiehlt sich daher, die Darstellung der Verbindung bei nur schwacher Belichtung vorzunehmen. Zur Analyse wurde die Substanz bei $100^{\circ}$ getrocknet. 
$0,1818 \mathrm{~g}$ Substanz gaben $0,4455 \mathrm{~g} \mathrm{CO}_{2}$ und $0,0855 \mathrm{~g} \mathrm{H}_{2} \mathrm{O}$. $0,2614 \mathrm{~g}$ Substanz gaben $29,25 \mathrm{ccm} N$ bei $17^{\circ}$ und $738 \mathrm{~mm}$ Druck. Berechnet für $\mathrm{C}_{18} \mathrm{H}_{17} \mathrm{~N}_{8} \mathrm{O}_{8}(323,2): 66,83 \% \mathrm{C}, 5,30 \% \mathrm{H}, 13,03 \% \mathrm{~N}$. Gefunden:

$66,83 \% \mathrm{C}, 5,26 \% \mathrm{H}, 12,80 \% \mathrm{~N}$.

$\beta$-Naphtalinsulfo-d-Tryptophannatrium.<smiles>CCCC(=O)C(CC1=CNC2CCCC12)NS(=O)(=O)c1ccccc1</smiles>

$1 \mathrm{~g}$ Tryptophan wurde in $5 \mathrm{ccm}$ Normalnatronlauge gelöst und mit einer ätherischen Lösung von 2,2 $\mathrm{g} \beta$-Naphtalin= sulfochlorid 3 Stunden auf der Maschine geschüttelt. Es wurden noch $3 \mathrm{mal}$ je $5 \mathrm{ccm}$ normaler Natronlauge in gleichmäßigen Intervallen hinzugegeben. Während des Schüttelns schied sich eine krystallinische Masse aus. Sie wurde abfiltriert und aus heißem Wasser umkrystallisiert. Sie bestand aus mikroskopischen Nadeln und zeigte den Schmelzpunkt $304^{\circ}$ (korr.). Beim Erhitzen auf dem Platinblech hinterblieb eine nicht verbrennbare Schmelze. Die Analyse stimmte auf $\mathrm{C}_{21} \mathrm{H}_{17} \mathrm{~N}_{2} \mathrm{O}_{4} \mathrm{SNa}$. Es lag daher das Natriumsalz des $\beta$-Naphtalinsulfotryptophans vor. Zur Analyse wurde die Substanz bei $125^{\circ}$ im Vakuum über Phosphorpentoxyd getrocknet.

$0,1715 \mathrm{~g}$ Substanz gaben $0,3804 \mathrm{~g} \mathrm{CO}_{2}$ und $0,0670 \mathrm{~g} \mathrm{H}_{2} \mathrm{O}$.

$0,1760 \mathrm{~g}$ Substanz gaben $10,5 \mathrm{ccm} N$ bei $16^{\circ}$ und $748 \mathrm{~mm}$ Druck. Berechnet für $\mathrm{C}_{21} \mathrm{H}_{17} \mathrm{~N}_{2} \mathrm{O}_{4} \mathrm{SNa}(416,3): 60,53 \% \mathrm{C}, 4,12 \% \mathrm{H}, 6,75 \% \mathrm{~N}$. Gefunden: $60,49 \% \mathrm{C}, 4,37 \% \mathrm{H}, 6,93 \% \mathrm{~N}$.

Wir halten dieses Derivat für sehr geeignet zur Isolierung des Tryptophans und werden, sobald wir noch weitere Erfahrungen besitzen, eingehender auf die Bestimmung des Tryptophans mit Hilfe dieser Verbindung zurückkommen.

Salzsaures d-Tryptophanchlorid.<smiles>NC(CC1CNC2CCCC12)C(=O)Cl</smiles>

2,4 g sehr sorgfältig gepulvertes Tryptophan wurden unter Eiskühlung mit $30 \mathrm{ccm}$ Acetylchlorid übergossen und mit $3,2 \mathrm{~g}$ 
Phosphorpentachlorid versetzt. Wir schüttelten erst 15 Minuten unter Kühlung mit Eiswasser, dann $11 / 2$ Stunden bei gewöhnlicher Temperatur. Dann wurde in der von E. Fischer ${ }^{1}$ ) beschriebenen Weise filtriert und erst mit Acetylchlorid, dann mit Petroläther gewaschen. Die abfiltrierte lockere Masse trockneten wir über Phosphorpentoxyd im Vakuum. Die Ausbeute betrug 3,0 g. Die Mutterlauge wurde nicht weiter verarbeitet, weil sie durch Verharzungsprodukte stark gefärbt war. Der isolierte Körper stellt das salzsaure Tryptophanchlorid dar. Zwar ergab die Analyse, daß der Körper etwa $1 \%$ Chlor zu wenig enthielt. Doch lag dies wahrscheinlich daran, daß die Darstellung im Hochsommer vorgenommen wurde, und die damals herrschende hohe Luftfeuchtigkeit auf das Präparat zersetzend gewirkt hatte. Der Körper gibt alle Reaktionen, die E. Fischer für die salzsauren Aminosäurechloride beschreibt. In Wasser löst er sich unter Erwärmung. Beim Abkühlen scheidet sich salzsaures Tryptophan aus der Lösung ab. In Methylalkohol geht er ebenfalls unter Wärmeentwicklung in Lösung. Durch Essigäther kann daraus Tryptophanmethylesterchlorhydrat von charakteristischer Krystallform und dem Schmelzpunkt $214^{\circ}$ (korr.) erhalten werden. Beim Erhitzen färbt sich die Substanz bei $172^{\circ}$, sintert bei etwa $208^{\circ}$ und schmilzt bei $228^{\circ}$ (korr.) unter Gasentwicklung.

Zur Analyse war die Substanz im Vakuum über Phosphorpentoxyd getrocknet. Sie wurde in Wasser gelöst und die abgespaltene Salzsäure titriert.

0,2075 g Substanz verbrauchten 15,63 ccm 1/10-normaler Silberlösung.

Berechnet für $\mathrm{C}_{11} \mathrm{H}_{12} \mathrm{~N}_{\mathbf{9}} \mathrm{OCl}_{\mathbf{2}}(259,1): 27,38 \% \mathrm{Cl}$.

Gefunden: $26,71 \% \mathrm{Cl}$.

Wir haben diese Verbindung zur Darstellung des Dipeptids Tryptophyl-glycin benützt. Die Beschreibung dieser Verbindung, sowie einiger anderer Polypeptide des Tryptophans erfolgt an anderer Stelle. ${ }^{2}$ )

1) E. Fischer, Synthese von Polypeptiden IX. Chloride der Aminosäuren. Ber. d. D. chem. Ges., Bd. XXXVIII, S. 605 (1905).

2) Berichte d. Deutsch. chem. Gesellsch., Jg. XL, 1907. 